\title{
QUERCETIN IMPROVES THE EFFICACY OF SORAFENIB IN TRIPLE NEGATIVE BREAST CANCER CELLS THROUGH THE MODULATION OF DRUG EFFLUX TRANSPORTERS EXPRESSIONS
}

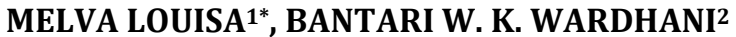

1Department of Pharmacology and Therapeutics, Faculty of Medicine, Universitas Indonesia, ${ }^{2}$ Doctoral Program in Biomedical Sciences, Faculty of Medicine, Universitas Indonesia Email: melva.louisa@gmail.com

Received: 14 Dec 2018, Revised and Accepted: 10 Mar 2019

\section{ABSTRACT}

Objective: This study aimed to investigate whether quercetin is able to improve the efficacy of sorafenib in triple negative breast cancer cells and explore the possibility of drug efflux transporters modulation by quercetin.

Methods: We exposed MDA-MB-231, a triple negative breast cancer cell line, to several groups: sorafenib alone, quercetin alone, a combination of sorafenib-quercetin, and control. We determined cell viability over control weekly up to $4 \mathrm{w}$. At the end of the fourth week, mRNA expressions of drug efflux transporters (P-glycoprotein and breast cancer resistance protein [BCRP] and MRP2 [multidrug resistance-associated protein-2]) were examined.

Results: Sorafenib alone was shown to maintain its efficacy for only two weeks, while quercetin alone was able to maintain its effect for four weeks. A combination of sorafenib-quercetin showed the best cytotoxicity effects compared with sorafenib or quercetin alone and was able to maintain its efficacy for four weeks. There were increased mRNA expressions of P-glycoprotein, BCRP, and MRP2 after four weeks of treatment with sorafenib, while treatment with quercetin decreased the drug efflux transporters expressions. A combination of sorafenib-quercetin decreased the mRNA expressions of both P-glycoprotein and BCRP, compared with sorafenib alone.

Conclusion: We suggest that decreased expressions of both drug efflux transporters, P-glycoprotein and BCRP, mediated by quercetin ameliorate the efficacy of sorafenib in TNBC. Therefore, the addition of quercetin to sorafenib might be useful in the future in improving the therapeutic efficacy of sorafenib in triple negative breast cancer.

Keywords: BCRP, MRP2, P-glycoprotein, Quercetin, Sorafenib

(C) 2019 The Authors. Published by Innovare Academic Sciences Pvt Ltd. This is an open access article under the CC BY license (http://creativecommons. org/licenses/by/4. 0/) DOI: http://dx.doi.org/10.22159/ijap.2019.v11s6.33576

\section{INTRODUCTION}

Breast cancer remains the most common type of malignancy for women around the world, including in the Asia-Pacific region, which accounts for $18 \%$ of all cancer diagnosed [1]. Despite the advances in early diagnosis and adjuvant treatment of this disease, many women still relapse with metastatic breast cancer [2].

Breast cancer is a heterogenous disease with diverse morphologies, molecular profiles, and responses to treatment [3]. About $10-20 \%$ of all breast cancers found are triple negative breast cancer (TNBC). TNBC is one particular type of cancer characterized by the lack of estrogen and progesterone receptors, as well as the absence of HER-2 protein expressions [3-5]. To date, TNBC is still an important clinical challenge, because these cancers do not respond to endocrine therapy or other available targeted agents [4]. Chemotherapy is still the treatment of choice for TNBC, though most patients do not achieve complete remission after chemotherapy. These problems have generated many studies into identifying an effective therapeutic strategy for TNBCs [5]. Current available treatment options for TNBC include anthracyclines, taxanes, platinum compounds, and some newer targeted therapy, including tyrosine kinase inhibitors [6].

One of the oral tyrosine kinase inhibitors used in breast cancer treatment is sorafenib. Sorafenib inhibits tumor growth and proliferation by interfering with several receptor tyrosine kinases involved in the pathogenesis of malignant breast cancer cells [7]. Unfortunately, an accumulating evidence showed that there was a tendency of increased acquired resistance to tyrosine kinase therapy. The known molecular mechanism of cancer cell resistance to tyrosine kinase inhibitors includes the mutation in kinase domains, epithelial mesenchymal transition, hypoxic microenvironment, and overexpression of $\mathrm{ABC}$ transporters [8].

Quercetin is a natural polyphenol that has been shown to exert anticancer activities in triple negative breast cancer cells through targeting the Akt/mammalian target of the rapamycin (mTOR) pathway in combination with the tyrosine kinase inhibitor, gefitinib [9]. Quercetin was also shown to regulate $\beta$-catenin signaling and downregulate vimentin in TNBC, thus it may potentiate existing chemotherapeutic drugs [10].

In the present study, we aimed to investigate whether quercetin might improve the therapeutic efficacy of sorafenib in triple negative breast cancers and to explore the possibility of drug efflux transporters modulation by quercetin.

\section{MATERIALS AND METHODS}

\section{Materials}

The MDA-MB-231 triple negative breast cancer cell line was a kind gift from the Tsukuba University Laboratory in Japan. Sorafenib was obtained from Santa Cruz; quercetin and DMSO were purchased from Sigma-Aldrich (Singapore). Dubelco Minimal Essential Medium (DMEM), Fetal Bovine Serum (FBS), penicillin/streptomycin, Fungizone, and Dubelco Phosphate Buffer Solution (D-PBS) were obtained from Biowest, USA. RNA isolation reagents, the Transcriptor First Strand cDNA Synthesis kit, and the FastStart DNA master SYBR Green I kit were purchased from Roche Diagnostics (Singapore); primers were obtained from $1^{\text {st }}$ BASE Ltd, Singapore.

\section{Cell cultures}

The MDA-MB-231 cells were cultured and maintained in DMEM supplemented with $10 \%$ heat-inactivated fetal bovine serum (FBS), 2 mmol L-glutamine, $100 \mathrm{IU} / \mathrm{ml}$ penicillin, $100 \mu \mathrm{g} / \mathrm{ml}$ streptomycin, and $1 \%$ Fungizone. The cells were subcultured when they reached about $90 \%$ confluency.

\section{MTS assay}

The MTS assay was used to determine the cytotoxicity concentration $50 \%$ of sorafenib and quercetin on MDA-MB-231. The CC50 found from this assay will be further used in drug treatment using 
sorafenib and quercetin. We applied sorafenib or quercetin three times in one week to obtain the repeated dose CC50.

\section{Drug treatment}

The MDA-MB-231 were plated at 10,000 cells per well and were treated with a medium containing sorafenib, quercetin, sorafenib and quercetin, or control (DMSO) in a 6-well plate. The sorafenib and quercetin were dissolved in DMSO at a final concentration of less than $0.001 \%$. Every week, the cells were trypsinized, counted, and 10,000 cells were re-plated in a new 6-well plate. Sorafenib with or without quercetin were given at the time of medium change, which was three times per week.

\section{Cell viability assay}

The cell viability assay was performed using the trypan blue exclusion method. The number of viable cells were counted using the trypan blue dye exclusion in a hemocytometer. The results were presented as the percentage of viable cells relative to the control group (DMSO).

\section{Quantitative reverse transcriptase PCR analysis}

The total cellular RNA was extracted from $1,000,000$ cells after trypsinization using the Total RNA Mini Kit (Geneaid, Taiwan) according to the manufacturer's protocol. Afterwards, $1 \mu \mathrm{g}$ of RNA was converted to cDNA using the Transcriptor First Strand cDNA Synthesis kit (Roche Diagnostics, Singapore).

The quantity and purity of the RNA and cDNA obtained were determined using NanoDrop Spectrophotometer (Thermo Scientific, USA). The cDNA obtained was then subjected to quantitative realtime reverse transcription polymerase chain reactions (qRT-PCR).

\section{qRT-PCR}

The mRNA expressions of the following drug transporters were quantified: P-glycoprotein, MRP2 (multidrug resistance protein-2), and BCRP (breast cancer resistance protein). A quantitative real-time reverse transcription polymerase chain reaction (qRT-PCR) was performed using the FastStart DNA master SYBR Green I kit (Roche, USA) on the Light Cycler Nano (Roche, USA) PCR Detection System. The following primers used were: P-glycoprotein, Fwd: TTACATTCAGGTTTCATTTTGGTG; Rev: TCCTGTCGCATTATAGCATGA; BCRP, Fwd: TTCGGCTTGCAACAACTATG; Rev: TCCAGACACAC CACGGATAA; MRP2, Fwd: ACAGAGGCTGGTGGCAACC; Rev: ACCAT TACCTTGTCACTGTCCATGA; $\beta$-actin (housekeeping gene): Fwd: GCTGGAAGGTGGACAGCGA, Rev GGCATCGTGATGGACTCCG

A total of $20 \mu \mathrm{l}$ reactions were performed in a 32-well plate on the qRT-PCR LightCycler Nano (Roche, USA). The RT-PCR conditions were set at a 3 -step amplification (45 cycles) in a specific annealing temperature of $52^{\circ}$ for P-glycoprotein and $\mathrm{BCRP}$, and $53^{\circ}$ for MRP2.

The quantification cycle (Cq) was analyzed by relative quantification using the Livak method and described as normalized expression ratios of P-glycoprotein, BCRP, and MRP2 to $\beta$-actin.

\section{Data analysis}

The data were presented in the form of means \pm standard deviation (SD). Graphs were created using GraphPad Prism software 7 (GraphPad, USA). The statistical significance was calculated using ANOVA One-Way, followed by a post hoc tests, with $\mathrm{p}<0.05$ considered as significant.

\section{RESULTS}

We obtained cytotoxicity concentrations of $0.6 \mu \mathrm{M}$ and $46.9 \mu \mathrm{M}$, respectively, for sorafenib and quercetin after repeated treatment in MDA-MB-231 (table 1). Based on the CC50 obtained after repeateddose administration, we used sorafenib at concentrations of $0.6 \mu \mathrm{M}$ and $0.3 \mu \mathrm{M}$, while for quercetin we used $25 \mu \mathrm{M}$ and $12.5 \mu \mathrm{M}$ for further experiments in MDA-MB-231.

Table 1: Cytotoxicity concentration $50 \%$ (CC50) of sorafenib and quercetin after repeated dose

\begin{tabular}{lc}
\hline & CC50 \\
\hline Sorafenib & $0.6 \mu \mathrm{M}$ \\
Quercetin & $46.9 \mu \mathrm{M}$ \\
\hline
\end{tabular}

Direct observation of MDA-MB-231 after four weeks of treatment did not cause visible changes in morphology, as seen throughout all treatment groups (fig. 1).

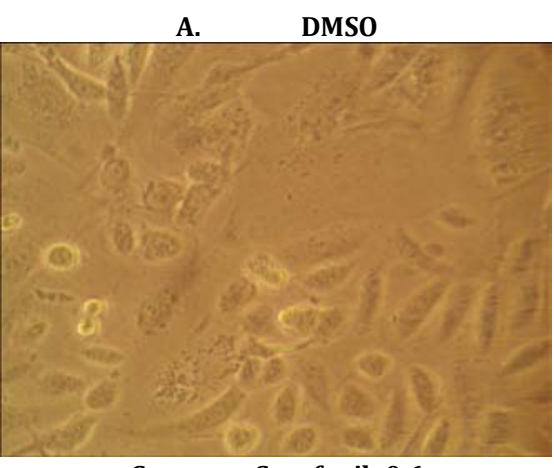

C. Sorafenib 0.6

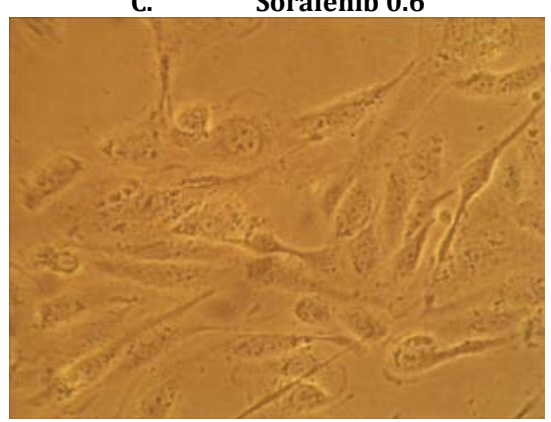

B. Sorafenib 0.3

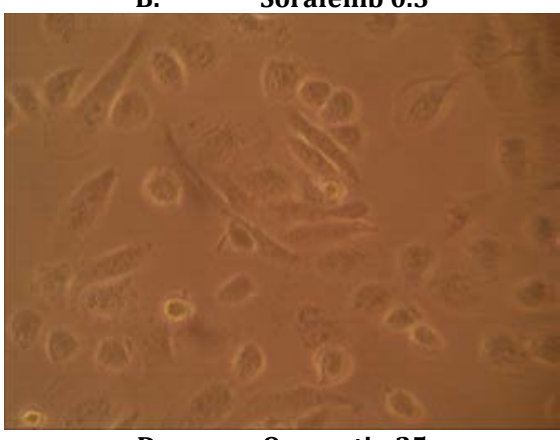

D.

Quercetin 25

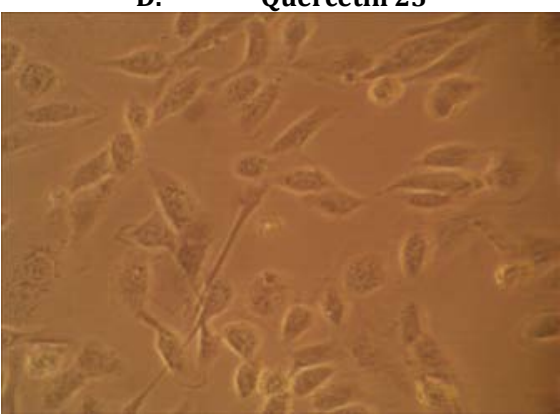



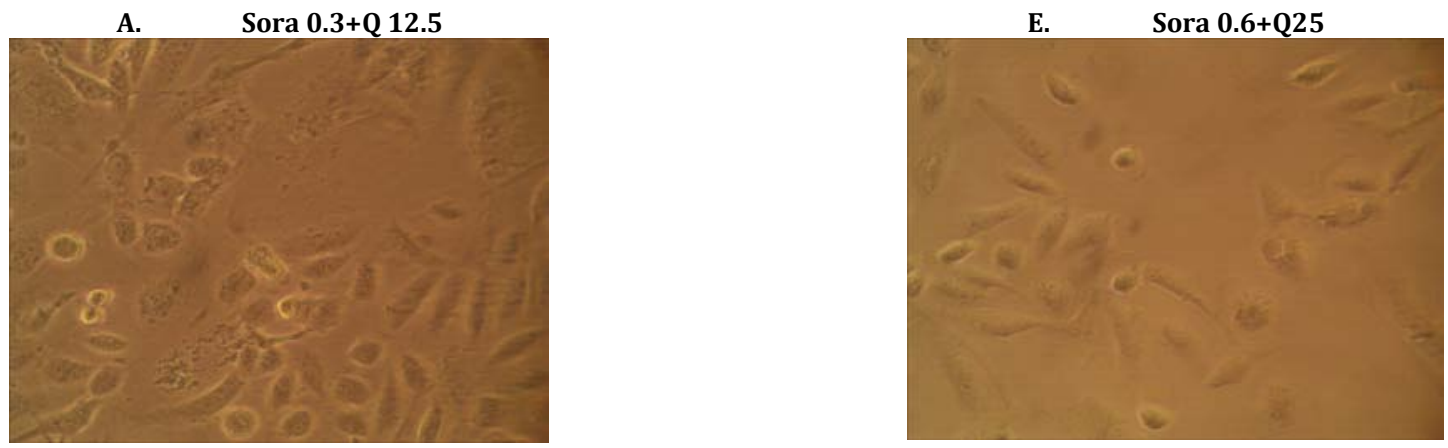

Fig. 1: Morphology of MDA-MB-231 cells after four weeks of treatment of A) control, B) quercetin $25 \mu \mathrm{M}, \mathrm{C}$ ) sorafenib $0.3 \mu \mathrm{M}, \mathrm{D}$ ) sorafenib $0.6 \mu \mathrm{m}$, E) sorafenib $0.3 \mu \mathrm{M}+$ quercetin $12.5 \mu \mathrm{M}$, sorafenib $0.6 \mu \mathrm{M}+$ quercetin $25 \mu \mathrm{M}$. Cells were observed using an inverted microscope at $20 \times$ magnification

After continuous treatment with for $4 \mathrm{w}$, it was shown that sorafenib alone was shown to maintain its efficacy for only two weeks, while quercetin alone was able to maintain its effect for four weeks. Fig. 2 showed that quercetin alone has a better cytotoxicity effect compared to the sorafenib-combination at weeks three and four. There was a marked increase of P-glycoprotein mRNA expressions in the sorafenib group and the effect was shown to be dose-dependent. Quercetin alone tended to decrease the expression of P-glycoprotein. A combination of sorafenib and quercetin at a higher dose significantly decreased Pglycoprotein expressions, but not in the lower dose (fig. 3).

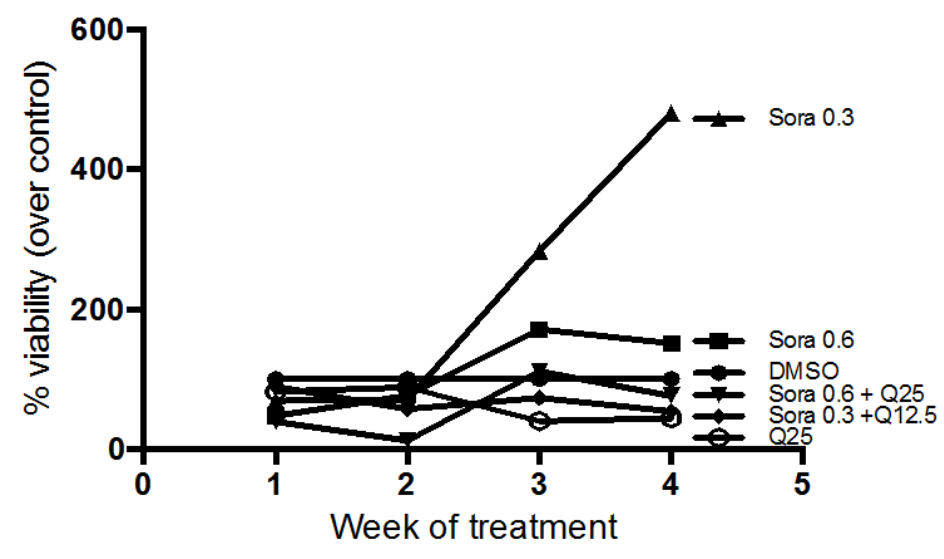

Fig. 2: Percentage of cell viability over control after four weeks of treatment with control (DMSO) or sorafenib $0.6 \mu \mathrm{M}$, sorafenib $0.3 \mu \mathrm{M}$, quercetin $25 \mu \mathrm{M}$, sorafenib $0.6 \mu \mathrm{M}+$ quercetin $25 \mu \mathrm{M}$, or sorafenib $0.3 \mu \mathrm{M}+$ quercetin $12.5 \mu \mathrm{M}$

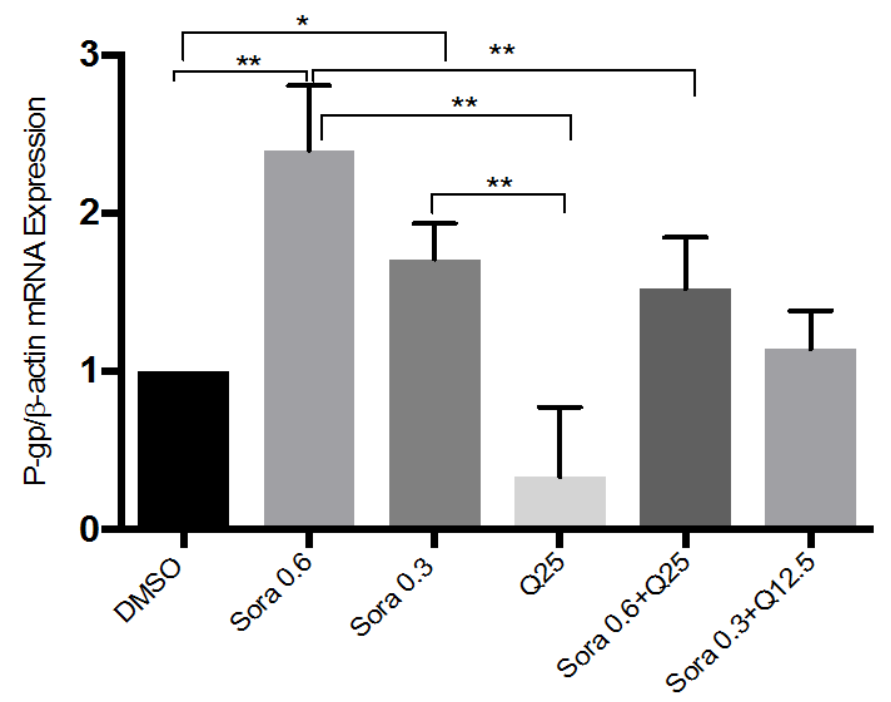

Fig. 3: mRNA expression of P-glycoprotein (normalized to $\beta$-actin) after four weeks of treatment with control (DMSO) or sorafenib $0.6 \mu M$, sorafenib $0.3 \mu \mathrm{M}$, quercetin $25 \mu \mathrm{M}$, sorafenib $0.6 \mu \mathrm{M}+$ quercetin $25 \mu \mathrm{M}$, or sorafenib $0.3 \mu \mathrm{M}+$ quercetin $12.5 \mu \mathrm{M}$, Data are expressed as means \pm SD of four experiments. *significantly different at $p<0.05$, ** significantly different at $p<0.001$ 
Increased BCRP mRNA expressions were shown in the sorafenib group and appeared to be dose-dependent. Quercetin alone resulted in a significant decrease of BCRP mRNA expressions. A combination of sorafenib and quercetin lowered BCRP expressions in sorafenib+quercetin groups versus sorafenib alone (fig. 4).

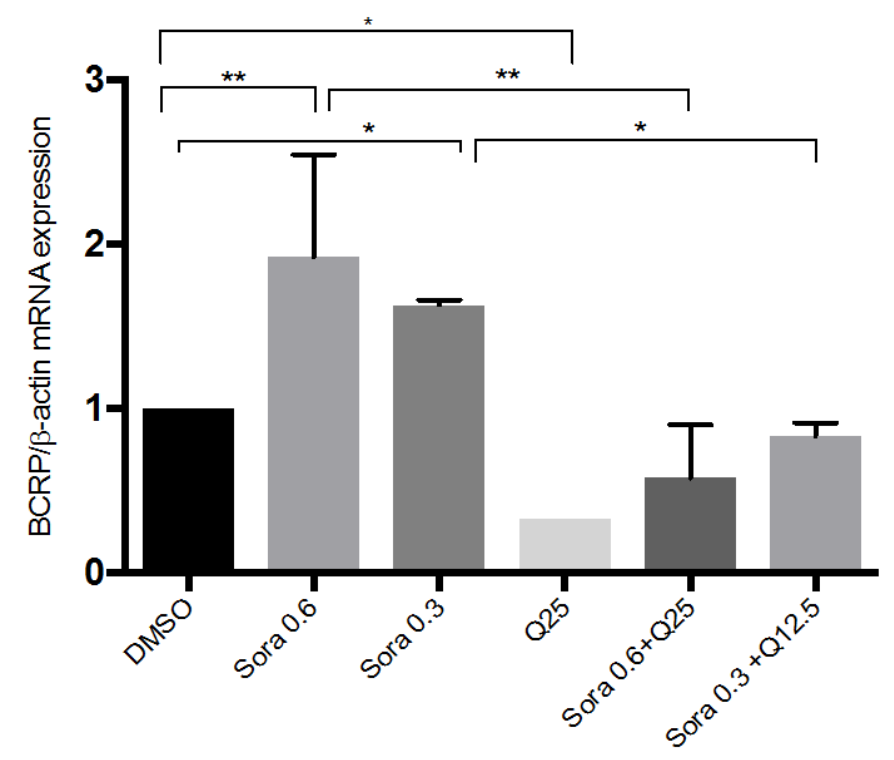

Fig. 4: mRNA expression of BCRP (normalized to $\beta$-actin) after four weeks of treatment with control (DMSO) or sorafenib $0.6 \mu \mathrm{m}$, sorafenib $0.3 \mu \mathrm{M}$, quercetin $25 \mu \mathrm{M}$, sorafenib $0.6 \mu \mathrm{M}$ +quercetin $25 \mu \mathrm{M}$, or sorafenib $0.3 \mu \mathrm{M}+$ quercetin $12.5 \mu \mathrm{m}$, Data are expressed as means $\square$ SD of four experiments. *significantly different at $\mathrm{p}<0.05,{ }^{* *}$ significantly different at $\mathrm{p}<0.001$

There was a marked increase in MRP2 mRNA expressions in the high dose sorafenib group. Quercetin tended to lower MRP2 expressions compared to the control. Lower MRP2 mRNA expressions were achieved in both sorafenib+quercetin groups versus sorafenib alone (fig. 5).

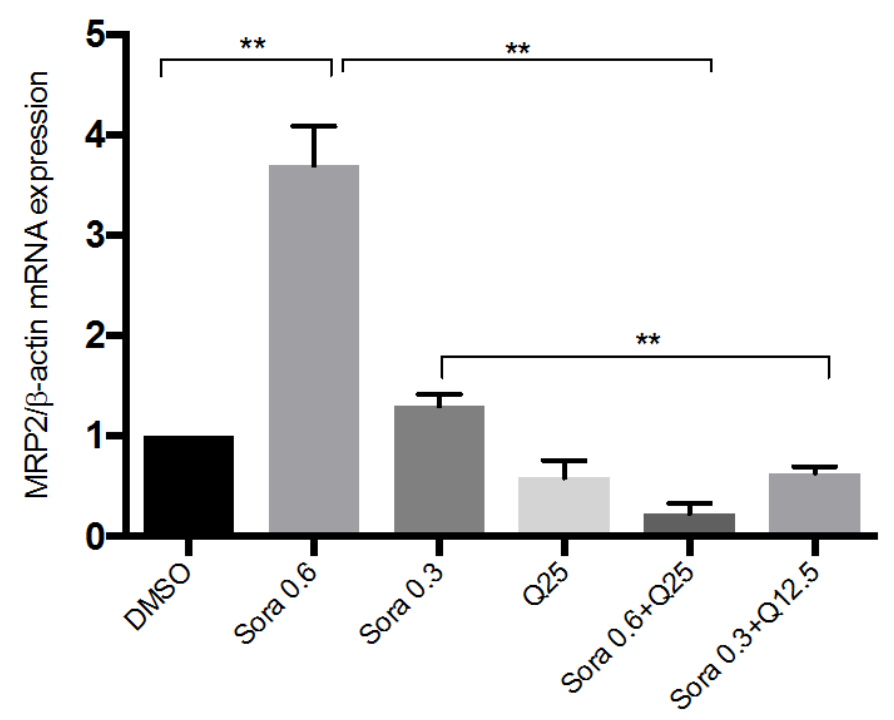

Fig. 5: mRNA expression of MRP2 (normalized to $\beta$-actin) after four weeks of treatment with control (DMSO) or sorafenib $0.6 \mu$ M, sorafenib $0.3 \mu \mathrm{M}$, quercetin $25 \mu \mathrm{M}$, sorafenib $0.6 \mu \mathrm{M}+$ quercetin $25 \mu \mathrm{M}$, or sorafenib $0.3 \mu \mathrm{M}+$ quercetin $12.5 \mu \mathrm{M}$, Data are expressed as means $\square$ SD of four experiments. *significantly different at $\mathrm{p}<0.05, * *$ significantly different at $\mathrm{p}<0.001$

\section{DISCUSSION}

Triple negative breast cancer is one of the most aggressive breast cancer types and is characterized by poor prognosis and a lack of treatment options [11]. Sorafenib is a multi-kinase inhibitor that has been used in clinical trial for breast cancer as a single agent or in combination with standard chemotherapy in advanced breast cancer [12-15]. Previous findings had confirmed that in metastatic breast cancer, single-agent sorafenib was not recommended due to the short time of the disease progression, [16] however, combinations of chemotherapeutic agents seemed promising [13] However, the place of sorafenib in the treatment of triple negative breast cancer has not yet been determined.

In our model of triple negative breast cancer cells, we found that sorafenib that was applied at CC50 had sufficient cytotoxicity effects 
only in the first two weeks of treatment; afterwards, the cancer cells quickly developed resistance towards sorafenib. Limited data are available explaining the mechanism of resistance of breast cancer cells to sorafenib. However, studies in hepatocellular carcinoma have revealed that several mechanisms may account for an acquired resistance to sorafenib, which includes overexpression of multi-drug resistance protein, that lead to the increased of drug efflux [17]. The combination of sorafenib and quercetin were shown to improve the cytotoxicity effect of sorafenib, while quercetin alone was able to steadily maintain its effects up to four weeks. To the best of our knowledge, this is the first to show a consistent effect of quercetin in a long-term application in triple negative breast cancer cells. Previous findings in triple negative breast cancer cells MDA-MB-231 and MDA-MB-468 using quercetin at concentrations ranging from $10-50 \mu \mathrm{M}$ in a shorter term of treatment (48 hour and 72 hour) proved that quercetin reduced the proliferation and viability of cells by regulating $\beta$-catenin signaling, inhibiting cell migration, altering vimentin and E-cadherin expression levels, and inhibiting the Akt/AMPK/mammalian target of rapamycin (mTOR) signaling cascade $[9,10]$.

Our results showed that sorafenib in all the doses tested increased P-glycoprotein mRNA expressions. This is in accordance with the previous study by Harmsen et al. in 2013, which reported that tyrosine kinase inhibitors, including sorafenib, were able to induce the expression of P-glycoprotein with a short period of $48 \mathrm{~h}$ in LS180 cells. They also showed that the upregulation of P-glycoprotein was mediated by pregnane X receptor (PXR) induction [18]. Previous studies on quercetin reported that quercetin is a potent inhibitor of P-glycoprotein expressions, [19] while in our results, though not significant, quercetin alone tended to decrease P-gp expressions. A combination of quercetin and sorafenib provided decreased expressions in P-glycoprotein compared with sorafenib alone. The results by Limtraku et al. (2005) also confirmed that quercetin and kaempferol inhibit the P-glycoprotein function in human cervical carcinoma KB-V1 cells (with high P-gp expressions) [20].

The above data suggested that sorafenib increased and quercetin strongly decreased BCRP mRNA expressions, while a combination of sorafenib and quercetin consistently lowered BCRP expressions. The study by Huang et al. in 2013 on short term treatment of sorafenib in hepatocellular carcinoma cells found that sorafenib leads to BCRP inhibition [21] Reports by Agarwal et al. [22] and Lagas et al.[23] state that sorafenib is a substrate of BCRP treatment [22,23]. Polyphenols (resveratrol, hesperetin, quervetin, daidzein, and silymarin) were shown to interact with BCRP in breast cancer cells of MCF7/MR (mitoxantrone resistant) and K562/BCRP cells, and this leads to increased accumulation of mitoxantrone in the cells [24].

Our data showed a marked increase in MRP2 mRNA expressions after long-term sorafenib treatment, and these expressions were lowered significantly in combination with quercetin. Our result is in agreement with Shibayama et al. (2011), who showed that sorafenib at CC50 increased MRP2 expressions by 6.4 times in renal cell carcinoma. That study also showed that sorafenib is a substrate for MRP2, suggesting that MRP2 may implicate drug resistance to sorafenib [17] The study by van Zanden et al. suggested that quercetin, or flavonoids in general, might worked as inhibitors to overcome MRP-mediated multidrug resistance [25]

Overall, our results indicate that quercetin is able to reduce the expressions of P-glycoprotein and also breast cancer resistance protein (BCRP) and MRP2 levels in TNBC cells. Further, the cellular upregulation of drug efflux transporters is also altered by quercetin treatment. Interestingly, quercetin alone was shown to maintain its cytotoxic effect in long-term treatment in triple negative breast cancer cells, which in our data might be due to the ability of quercetin to maintain a low expression of drug efflux transporters. Study by Srinivasan et al. [26] has also confirmed that quercetin is capable of inhibiting chemotherapy-induced TGF- $\beta$ signaling in TNBC cells, and that it would decrease the development of chemotherapy resistance in TNBC. Further, quercetin is able to inhibit the enhanced migration and mammosphere formation in chemoresistant TNBC cells [26]. Taken together, our findings revealed that quercetin is a promising natural product that increases the anti-cancer effect of chemotherapy drugs in TNBC. Further studies in animal models are needed to confirm the therapeutic effect of quercetin in TNBC.

\section{CONCLUSION}

In conclusion, we suggest that decreased expressions of both drug efflux transporters mediated by quercetin ameliorate the efficacy of sorafenib in TNBC. Therefore, the addition of quercetin to sorafenib might have be useful in the future in improving the therapeutic efficacy of sorafenib in triple negative breast cancer.

\section{ACKNOWLEDGEMENT}

This article was presented at The $3^{\text {rd }}$ International Conference and Exhibition on Indonesian Medical Education and Research Institute (ICE on IMERI 2018), Faculty of Medicine, Universitas Indonesia, Jakarta, Indonesia. This study was funded by the Ministry of Research Technology and Higher Education Research Grant. We thank the $3^{\text {rd }}$ ICE on IMERI Committee who had supported the peer review and manuscript preparation before submitting to the journal.

\section{AUTHORS CONTRIBUTIONS}

All the author have contributed equally

\section{CONFLICT OF INTERESTS}

We declare that we have no conflict of interest

\section{REFERENCES}

1. Youlden DR, Cramb SM, Yip CH, Baade PD. Incidence and mortality of female breast cancer in the Asia-pacific region. Cancer Biol Med 2014;11:101-15.

2. Sankaranarayanan R, Ramadas K, Qiao Y. Managing the changing burden of cancer in Asia. BMC Med 2014;12:3.

3. Bosch A, Eroles P, Zaragoza R, Vina JR, Lluch A. Triple-negative breast cancer: Molecular features, pathogenesis, treatment and current lines of research. Cancer Treat Rev 2010;36:206-15.

4. O'Reilly EA, Gubbins L, Sharma S. The fate of chemoresistance in triple negative breast cancer (TNBC). BBA Clin 2015;3:257-75.

5. Kalimutho M, Parsons K, Mittal D. Targeted therapies for triplenegative breast cancer: combating a stubborn disease. Trends Pharmacol Sci 2015;36:822-46.

6. Wahba HA, El-Hadaad HA. Current approaches in treatment of triple-negative breast cancer. Cancer Biol Med 2015;12:106-16.

7. Moreno Aspitia A. Clinical overview of sorafenib in breast cancer. Future Oncol 2010;6:655-63.

8. Chen Y, Fu L. Mechanisms of acquired resistance to tyrosine kinase inhibitors. Acta Pharm Sin B 2011;1:197-207.

9. Rivera AR, Castillo Pichardo L, Gerena Y. Anti-breast cancer potential of quercetin via the Akt/AMPK/Mammalian target of rapamycin (mTOR) signaling cascade. PLoS One 2016;11:e0157251.

10. Srinivasan A, Thangavel C, Liu Y. Abstract 5560: quercetin overcomes chemotherapy resistance in triple negative breast cancer. Cancer Res 2015;75:5560.

11. Engebraaten 0, Vollan HKM, Børresen-Dale AL. Triple-negative breast cancer and the need for new therapeutic targets. Am J Pathol 2013;183:1064-74.

12. Baselga J, Costa F, Gomez H. A phase 3 trial comparing capecitabine in combination with sorafenib or placebo for treatment of locally advanced or metastatic HER2-negative breast cancer (the RESILIENCE study): study protocol for a randomized controlled trial. Trials 2013;14:228.

13. Chen J, Tian CX, Yu M. Efficacy and safety profile of combining sorafenib with chemotherapy in patients with HER2-negative advanced breast cancer: a meta-analysis. J Breast Cancer 2014;17:61-8.

14. Ferrario C, Strepponi I, Esfahani K. Phase I/II Trial of sorafenib in combination with vinorelbine as first-line chemotherapy for metastatic breast cancer. PloS One 2016;11:e0167906.

15. Moreno Aspitia A, Morton RF, Hillman DW. Phase II trial of sorafenib in patients with metastatic breast cancer previously exposed to anthracyclines or taxanes: North central cancer treatment group and mayo clinic trial N0336. J Clin Oncol 2009;27:11-5. 
16. Bianchi G, Loibl S, Zamagni C. Phase II multicenter, uncontrolled trial of sorafenib in patients with metastatic breast cancer. Anticancer Drugs 2009;20:616-24.

17. Shibayama Y, Nakano K, Maeda H. Multidrug resistance protein 2 implicates anticancer drug-resistance to sorafenib. Biol Pharm Bull 2011:34:433-5.

18. Harmsen S, Meijerman I, Maas Bakker RF. PXR-mediated Pglycoprotein induction by small molecule tyrosine kinase inhibitors. Eur J Pharm Sci 2013;48:644-9.

19. Borska S, Chmielewska M, Wysocka T. In vitro effect of quercetin on human gastric carcinoma: targeting cancer cells death and MDR. Food Chem Toxicol 2012;50:3375-83.

20. Limtraku P, Khantamat O, Pintha K. Inhibition of P-glycoprotein function and expression by kaempferol and quercetin. J Chemother 2005; 17:86-95.

21. Huang WC, Hsieh YL, Hung CM. BCRP/ABCG2 inhibition sensitizes hepatocellular carcinoma cells to sorafenib. PLoS One 2013;8:e83627.
22. Agarwal S, Sane R, Ohlfest JR, Elmquist WF. The role of the breast cancer resistance protein (ABCG2) in the distribution of sorafenib to the brain. J Pharmacol Exp Ther 2011;336:223-33.

23. Lagas JS, Van Waterschoot RAB, Sparidans RW. Breast cancer resistance protein and P-glycoprotein limit sorafenib brain accumulation. Mol Cancer Ther 2010; 9:319-26

24. Zhang S, Yang X, Morris ME. Flavonoids are inhibitors of breast cancer resistance protein (ABCG2)-mediated transport. Mol Pharmacol 2004;65:1208-16

25. Van Zanden JJ, Van der Woude H, Vaessen J. The effect of quercetin phase II metabolism on its MRP1 and MRP2 inhibiting potential. Biochem Pharmacol 2007;74:345-51.

26. Srinivasan A, Thangavel C, Liu Y. Quercetin regulates $\beta$-catenin signaling and reduces the migration of triple negative breast cancer. Mol Carcinog 2016;55:743-56. 